\title{
M-strings and transverse orbifold
}

\author{
Amer Iqbal ${ }^{1, a}$, Khurram Shabbir ${ }^{2, b}$ \\ ${ }^{1}$ Abdus Salam School of Mathematical Sciences, Government College University, Lahore, Pakistan \\ ${ }^{2}$ Department of Mathematics, Government College University, Lahore, Pakistan
}

Received: 30 August 2016 / Accepted: 20 October 2016 / Published online: 8 November 2016

(c) The Author(s) 2016. This article is published with open access at Springerlink.com

\begin{abstract}
We discuss the partition function of a single M5brane on a circle with transverse orbifold of ADE type and show that the modes captured by the partition function are those of the tensor multiplet and the three form field. We show that the bound states of M-strings corresponding to pair of simple roots appear, for all $\mathrm{ADE}$, only when the momentum on the circle is turned on.
\end{abstract}

\section{Contents}

1 Introduction ................. 1

2 M5-brane configuration and its partition function .. 1

2.1 Partition function and Hilbert scheme of points 2

2.2 Modes and supermultiplets . . . . . . . . . 3

2.2.1 Single M5-brane . . . . . . . . . . . 3

2.2.2 M5-brane with transverse orbifold and modes 3

3 ADE orbifolds and bound states of M-strings . . . . 4

4 Conclusions .............. . . 5

Appendix A . . . . . . . . . . . . 5

References ............... 6

\section{Introduction}

In this paper we study the partition function of an M5-brane on a circle with transverse space $S^{1} \times \mathbb{C}^{2} / \Gamma$ in the limit the circle size becomes large. This brane configuration has a dual Calabi-Yau geometry which is toric for the case when $\Gamma=\mathbb{Z}_{k}$ i.e., $A$ type orbifold [1,2]. For the A type orbifold the partition function can be determined using the refined topological vertex. Interestingly in the toric case $\Gamma=\mathbb{Z}_{k}$ this configuration is dual to the configuration in which we can get rid of the transverse orbifold at the expense of having $k$ M5branes wrapped on the circle. This duality can be achieved

\footnotetext{
a e-mail: amer@alum.mit.edu

be-mail: dr.khurramshabbir@gcu.edu.pk
}

by going to string theory and using T-duality which maps $A$ type orbifold to NS5-branes. As was discussed in $[3,4]$ this can also be understood in terms of T-duality of little string theory.

In the case when the transverse space was just $\mathbb{R}^{5}$ it was shown in [5] that refined topological string partition function of a certain toric elliptic Calabi-Yau threefold captures the field content completely which was just the circle reduction of the $(2,0)$ tensor multiplet. We discuss in detail the case when the space transverse to the M5-brane is $S^{1} \times \mathbb{C}^{2} / \Gamma_{A D E}$, where $\Gamma_{A D E}$ is the ADE discrete subgroup of $S U(2)$. We show that in the case $\Gamma_{A D E}=\mathbb{Z}_{k}$ there is corresponding toric elliptically fibered CY3fold whose refined topological string partition function gives the modes of the $(2,0)$ tensor multiplet as well as the modes coming of the M-theory three form field reduced on the circle and the orbifold. We also show that for all ADE orbifolds M-strings do not form bound states unless the momentum is turned on the circle on which the M5-brane is wrapped.

This paper is organized as follows. In Sect. 2 we discuss the Calabi-Yau geometries dual to brane configuration shown in Fig. 1 and give the corresponding partition function for the $A$ type orbifold. In this section we also give the dual form of the partition function coming from its realization as $\chi_{y}$ genus of product of Hilbert scheme of points on $\mathbb{C}^{2}$. In Sect. 3 we discuss the mode expansion of the refined partition functions and show that they capture the tensor multiplet modes as well as those coming from the three form field. In Sect. 4 we generalize the A type partition function to $D$ and $E$ type and discuss the appearance of bound states when momentum is turned on. In Sect. 4 we discuss our conclusions and some work in progress.

\section{M5-brane configuration and its partition function}

In this section we will discuss the calculation of the partition function of the a single M5-brane with transverse space $S^{1} \times \mathbb{C}^{2} / \mathbb{Z}_{k}$. Going down to type IIB and using T-duality 


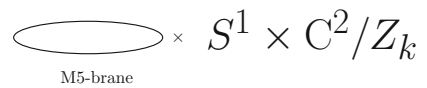

(a)

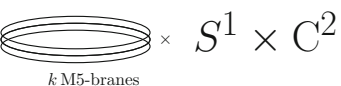

(b)
Fig. 1 Dual brane configurations

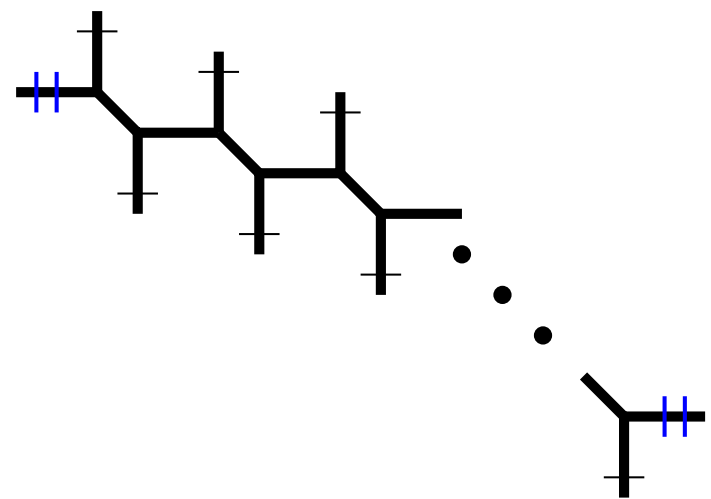

Fig. 2 a $k$ M5-branes on a circle with transverse space $S^{1} \times \mathbb{C}^{2} / \Gamma$. b The dual Calabi-Yau geometry after resolving the singularities of the transverse orbifold

this brane/geometry configuration can be mapped to an intersecting system of a single D5-brane and $k$ NS5-branes. The $S L(2, \mathbb{Z})$ symmetry of type IIB can then map $1 \mathrm{D} 5+k$ NS-5 to $1 \mathrm{NS}-5+k$ D5, which can then be lifted back to M-theory to get $k$ M5-branes with transverse space $S^{1} \times \mathbb{C}^{2}$ establishing the duality of Fig. 1. If we resolve the D5-brane/NS5-brane intersections we obtain a brane web as shown in Fig. 2.

As is well known the $(p, q) 5$-brane webs are dual to Calabi-Yau threefolds [6] and in this case there is a double elliptically fibered Calabi-Yau threefolds dual to the above brane configuration $[3,4]$. This threefold is actually a $\mathbb{Z}_{k}$ orbifold of the threefold corresponding to $k=1$. The $k=1$ threefold is a double elliptic fibration over the complex plane with a single $I_{0}$ fiber of both fibrations. In the limit when we take the circle on which the M5-brane is wrapped to infinity this threefold for the $\mathbb{Z}_{k}$ case is birationally equivalent to $\widehat{A}_{k-1} \times{ }_{f} \mathbb{C}$ where $\widehat{A}_{k-1}$ is affine $A_{k-1}$ space blown up at $k$ points and it is fibered over $\mathbb{C}$ to obtain the Calabi-Yau threefold.

The affine $A_{k-1}$ space has $k \mathbb{P}^{1}$ 's corresponding to the simple roots. We denote these curve classes by $C_{a}$ with $a=1,2, \ldots, k$. The blow-up introduces $k$ new curve classes which we denote $M_{a}$ with $a=1,2, \ldots, k$. The class of the elliptic curve $E$ is given by

$E=C_{1}+C_{2}+\cdots+C_{k}$.

The complexified Kähler parameters associated with these curves are given by the Kähler form $\omega$ :

$t_{a}=\int_{C_{a}} \omega, \quad \rho=\int_{E} \omega, m=\int_{M_{a}} \omega, a=1,2, \ldots, k$.
Because of Eq. (1) $\rho$ is given by

$\rho=t_{1}+t_{2}+\cdots+t_{k}$.

The topological string partition function can be calculated using the refined topological vertex [7] and is given by [8]:

$Z_{k}:=\sum_{v} \prod_{a=0}^{k-1}\left[\left(-Q_{a}\right)^{\left|\nu_{(a)}\right|} G_{\nu_{(a)} \nu_{(a+1)}}\right]$

where

$G_{\nu_{(a)} \nu_{(a+1)}}=\sum_{\mu}\left(-Q_{m}\right)^{|\mu|} C_{\lambda_{(a)}^{t} \mu \emptyset}(t, q) C_{\lambda_{(a+1)} \mu^{t} \emptyset}(q, t)$

and

$$
\begin{aligned}
C_{\lambda \mu \emptyset}(t, q)= & \left(\frac{q}{t}\right)^{\frac{|\lambda|+\left.|| \mu\right|^{2}-|\mu|}{2}} t^{\frac{\kappa(\mu)}{2}} \widetilde{Z}_{v}(t, q) \\
& \times \sum_{\eta}\left(\frac{q}{t}\right)^{\frac{|\eta|}{2}} s_{\lambda^{t} / \eta}\left(t^{-\rho}\right) s_{\mu / \eta}\left(q^{-\rho}\right)
\end{aligned}
$$

is the refined topological vertex (see Appendix A for notation and conventions). The length of the slanted lines in Fig. 2 are all equal to $m$ and we have defined $Q_{m}=e^{2 \pi i m}$, similarly the length of the horizontal lines is $T_{a}$ and we have defined $Q_{a}=e^{2 \pi i T_{a}}$ such that $t_{a}=\log \left(Q_{a} Q_{m}\right) / 2 \pi i$ is the distance between the two vertical lines. Using the identity

$\sum_{\lambda \eta} Q_{\rho}^{|\lambda|} s_{\lambda^{t} / \eta}(x) s_{\lambda / \eta}(y)=\prod_{k=1}^{\infty}\left(1-Q_{\rho}^{k}\right)^{-1} \prod_{i, j}\left(1-Q_{\rho}^{k} x_{i} y_{j}\right)$

we can calculate the sum in Eq. (3) to obtain

$$
\begin{aligned}
& Z_{k}\left(\rho, m, \mathbf{t}, \epsilon_{1,2}\right)=Z_{1}^{k}\left(\prod_{n=1}^{\infty}\left(1-Q_{\rho}^{n}\right)\right)^{k-1} \\
& \times \prod_{1 \leq a<b \leq k} \frac{G_{2}\left(Q_{a b} ; \rho, \epsilon_{1}, \epsilon_{2}\right) G_{2}\left(Q_{\rho} Q_{a b}^{-1} ; \tau, \epsilon_{1}, \epsilon_{2}\right)}{G_{2}\left(Q_{a b} Q_{m} \sqrt{t q} ; \rho, \epsilon_{1}, \epsilon_{2}\right) G_{2}\left(Q_{a b} Q_{m}^{-1} \sqrt{t q} ; \rho, \epsilon_{1}, \epsilon_{2}\right)}, \\
& Z_{1}\left(\rho, m, \epsilon_{1,2}\right)=\prod_{i, j, k=1}^{\infty} \\
& \times \frac{\left(1-Q_{\rho}^{k-1} Q_{m} q^{i-\frac{1}{2}} t^{j-\frac{1}{2}}\right)\left(1-Q_{\rho}^{k} Q_{m}^{-1} q^{i-\frac{1}{2}} t^{j-\frac{1}{2}}\right)}{\left(1-Q_{\rho}^{k} q^{i} t^{j}\right)\left(1-Q_{\rho}^{k} q^{i-1} t^{j-1}\right)} \\
& G_{2}\left(x ; \rho, \epsilon_{1}, \epsilon_{2}\right)=\prod_{k, i, j=1}^{\infty} \\
& \times\left(1-Q_{\rho}^{k-1} q^{i-1} t^{-j+1} x\right)\left(1-Q_{\rho}^{k} q^{i} t^{-j} x^{-1}\right),
\end{aligned}
$$

where $Q_{a b}=\exp \left(2 \pi i\left(t_{b}-t_{a}\right)\right)$.

\subsection{Partition function and Hilbert scheme of points}

The above partition function that we obtained in Eq. (5) related to the index of Dirac operator couple to bifundamental matter and can be obtained using a slight generalization 
of the $\chi_{y}$ genus. Recall that the $\chi_{y}$ genus of a manifold $M$ is given by

$\chi_{y}(M)=\int_{M} \prod_{i} \frac{x_{i}\left(1-y e^{-x_{i}}\right)}{1-e^{-x_{i}}}$,

where $x_{i}$ are roots of the Chern polynomial of the tangent bundle of $M$. It can be generalized using a vector bundle $V$ of rank equal to the rank of the tangent bundle to get

$\chi_{y}(M, V)=\int_{M} \prod_{i} \frac{x_{i}\left(1-y e^{-w_{i}}\right)}{1-e^{-x_{i}}}$,

where $w_{i}$ are the roots of the Chern polynomial of $V$. Using $\chi_{y}(M, V)$ we can write the partition function in Eq. (5) as

$Z_{k}=\sum_{m_{1}, m_{2}, \ldots, m_{k}} Q_{1}^{m_{1}} \ldots Q_{k}^{m_{k}} \chi_{Q_{m}}\left(H_{m_{1}, \ldots, m_{k}}, V\right)$,

where $H_{m_{1}, \ldots, m_{k}}$ is the product of the Hilbert scheme of points on $\mathbb{C}^{2}$,

$H_{m_{1}, \ldots, m_{k}}=\operatorname{Hilb}^{m_{1}}\left[\mathbb{C}^{2}\right] \times \operatorname{Hilb}^{m_{2}}\left[\mathbb{C}^{2}\right] \times \cdots \times \operatorname{Hilb}^{m_{k}}\left[\mathbb{C}^{2}\right]$,

and $V$ is a vector bundle on $H_{m_{1}, \ldots, m_{k}}$ whose fiber at a point can be described as follows. Recall that the $\mathrm{Hilb}^{m}\left[\mathbb{C}^{2}\right]$ is the resolution of $m$ th symmetric product of $\mathbb{C}^{2}$ and points in it can be identified with codimension $m$ ideals in the ring $\mathbb{C}[x, y]$ [9]. Thus a point in $H_{m_{1}, \ldots, m_{k}}$ is a collection of ideals $\left(I_{1}, I_{2}, \ldots, I_{k}\right)$ such that $I_{a} \subset \mathbb{C}[x, y]$ and $\operatorname{dim}\left(\mathbb{C}[x, y] / I_{a}\right)=m_{a}$. The fiber of the vector bundle $V$ over the point $\left(I_{1}, I_{2}, \ldots, I_{k}\right)$ is given by

$\operatorname{Ext}^{1}\left(I_{1}, I_{2}\right) \oplus \operatorname{Ext}^{1}\left(I_{2}, I_{3}\right) \oplus \cdots \oplus \operatorname{Ext}^{1}\left(I_{k}, I_{1}\right)$.

For the ADE case it is natural to expect that the partition function is still given by an expression of the kind of Eq. (8) with manifold

$\mathcal{M}\left(r_{1}, m_{1}\right) \times \mathcal{M}\left(r_{2}, m_{2}\right) \times \cdots \times \mathcal{M}\left(r_{k}, m_{k}\right)$,

where $\mathcal{M}(r, m)$ is the moduli space of $U(r)$ instantons on $\mathbb{C}^{2}$ with charge $m$ and $r_{a}$ are the Kac labels of the ADE Dynkin diagram. The description of the bundle in this case is slightly more involved and will be discussed in [10].

\subsection{Modes and supermultiplets}

From the partition function of Eq. (5) we can determine the contribution of various supermultiplets in five dimensions. Massless particles in five dimensions have little group $\operatorname{Spin}(3)=S U(2)$ and the massive particles have little group $\operatorname{Spin}(4)=S U(2)_{L} \times S U(2)_{R}$. Fields in the massive multiplet (which is actually a $(2,0)$ multiplet of the $\mathcal{N}=2$ supersymmetry in five dimensions) are in the following representation [11]:

$$
(1,0) \oplus 4\left(\frac{1}{2}, 0\right) \oplus 5(0,0) \text {. }
$$

The five dimensional vector multiplet containing the massless vector field is in the following representation of Spin(3) [11]:

(1) $\oplus 4\left(\frac{1}{2}\right) \oplus 5(0)$.

\subsubsection{Single M5-brane}

The case of a single M5-brane (i.e., $k=1$ ) was discussed in $[5,12]$. We discuss it briefly to make comparison later to $k>1$ case. From the plethystic logarithm of the $Z_{1}$ in Eq. (5) we get

$$
\begin{aligned}
& F_{1}=P \log \left(Z_{1}\right), \\
& F_{1}+\overline{F_{1}}=\underbrace{Q_{m}+Q_{m}^{-1}-\sqrt{\frac{q}{t}-\sqrt{\frac{t}{q}}}+}_{\text {massless }} \\
& \times \sum_{k \in \mathbb{Z}, k \neq 0}^{\infty} \underbrace{Q_{\tau}^{k}\left[\left(Q_{m}+Q_{m}^{-1}\right)-\left(\sqrt{q t}+\frac{1}{\sqrt{q t}}\right)\right]}_{\text {massive }} .
\end{aligned}
$$

In the above equation we have indicated the massless and the KK massive modes. From Eq. (13) it follows that massless and the massive multiplets are in the following representation of the $S U(2)_{L} \times S U(2)_{R}$ :

massless: $\quad\left(0, \frac{1}{2}\right) \oplus 2(0,0)$,

massive: $\left(\frac{1}{2}, 0\right) \oplus 2(0,0)$.

As discussed in [12] the contribution of the universal half hypermultiplet is needed to get the full Spin(4) content [13]. The half hypermultiplet is $\left(\frac{1}{2}, 0\right) \oplus 2(0,0)$ and tensoring with it and reducing to the diagonal for the massless gives $[5,12]$

massless: $\quad(1) \oplus 4\left(\frac{1}{2}\right) \oplus 5(0)$,

massive: $\quad(1,0) \oplus 4\left(\frac{1}{2}, 0\right) \oplus 5(0,0)$.

The above is the six dimensional tensor multiplet reduced on the circle [11].

\subsubsection{M5-brane with transverse orbifold and modes}

For the case of M5-brane with transverse orbifold we use $Z_{k}$ given in Eq. (5). To determine the spin content of the various modes we consider $F_{k}+\overline{F_{k}}$ where $F_{k}$ is defined by

$Z_{k}=\exp \left(-\sum_{n=1}^{\infty} \frac{F_{k}\left(n \rho, n m, n \epsilon_{1}, n \epsilon_{2}\right)}{n\left(q^{\frac{n}{2}}-q^{-\frac{n}{2}}\right)\left(t^{\frac{n}{2}}-t^{-\frac{n}{2}}\right)}\right)$. 
Using the identity

$G_{2}\left(x ; \rho, \epsilon_{1},-\epsilon_{2}\right)=P E\left(-\frac{x+x^{-1} Q_{\rho} q t}{\left(1-Q_{\rho}\right)(1-q)(1-t)}\right)$,

where $P E$ denotes the plethystic exponential, we get

$$
\begin{aligned}
F_{k}+\overline{F_{k}}= & k\left(Q_{m}+Q_{m}^{-1}-\sqrt{\frac{t}{q}}-\sqrt{\frac{q}{t}}\right) \\
& +\sum_{n \in \mathbb{Z}, n \neq 0} Q_{\rho}^{n}\left[k\left(Q_{m}+Q_{m}^{-1}\right)\right. \\
& \left.-\sqrt{t q}-\frac{1}{\sqrt{t q}}-(k-1)\left(\sqrt{\frac{t}{q}}+\sqrt{\frac{q}{t}}\right)\right] \\
& +\sum_{1 \leq a<b \leq k} \sum_{n \in \mathbb{Z}} Q_{\rho}^{n}\left(Q_{a b}+Q_{a b}^{-1}\right) \\
& \times\left(Q_{m}+Q_{m}^{-1}-\sqrt{t q}-\frac{1}{\sqrt{t q}}\right) .
\end{aligned}
$$

From the above we see that the massless and the massive multiplets are in the following representations of $S U(2)_{L} \times$ $S U(2)_{R}$ :

$$
\begin{aligned}
\text { massless: } & {\left[2(0,0) \oplus\left(0, \frac{1}{2}\right)\right] \oplus(k-1)\left[2(0,0) \oplus\left(0, \frac{1}{2}\right)\right], } \\
\text { massive: } & {\left[2(0,0) \oplus\left(\frac{1}{2}, 0\right)\right] \oplus(k-1)\left[2(0,0) \oplus\left(0, \frac{1}{2}\right)\right] } \\
& \oplus \frac{k(k-1)}{2}\left[2(0,0) \oplus\left(\frac{1}{2}, 0\right)\right] .
\end{aligned}
$$

As discussed for the $k=1$ case we are still missing the contribution of the universal half hypermultiplet associated with the position of the particle in $\mathbb{R}^{4}$. Tensoring with the half hypermultiplet $2(0,0) \oplus\left(\frac{1}{2}, 0\right)$ gives

$$
\begin{aligned}
& \text { massless: }[\underbrace{(1) \oplus 4\left(\frac{1}{2}\right)+5(0)}_{\text {tensor multiplet }}] \oplus(k-1)[\underbrace{(1) \oplus 4\left(\frac{1}{2}\right)+5(0)}_{\text {massless veto }}] \\
& \text { massive: }[\underbrace{(1,0) \oplus 4\left(\frac{1}{2}, 0\right) \oplus 5(0,0)}_{\text {tensor multiplet }}] \\
& \oplus(k-1)[\underbrace{\left(\frac{1}{2}, \frac{1}{2}\right) \oplus 2\left(0, \frac{1}{2}\right) \oplus 2\left(\frac{1}{2}, 0\right) \oplus 4(0,0)}_{\text {massive vector multiplet }}] \\
& \oplus \underbrace{\oplus \frac{k(k-1)}{2}\left[(1,0) \oplus 4\left(\frac{1}{2}, 0\right) \oplus 5(0,0)\right]}_{\text {BPS State for each positive root }} .
\end{aligned}
$$

Thus we see that the supermultiplets correspond to reduction of one $6 \mathrm{D}$ tensor multiplet on a circle together with $(k-1)$ vector multiplets coming from the three form field which we can write as

$C^{(3)}=\sum_{a=1}^{k-1} A^{a} \wedge \omega^{a}$, where $A^{a}$ is the gauge field of the vector multiplet and $\omega^{a}$ are the Kähler form for the curves corresponding to simple positive roots in the resolved orbifold.

\section{ADE orbifolds and bound states of M-strings}

In the previous sections we discussed the partition function of an M5-brane with transverse $A_{N}$ geometry. This partition function can be obtained in many different ways using the duality of this brane configuration with Calabi-Yau geometry, 2D gauge theories or 2D sigma models. For the case when the transverse orbifold is of $D$ or $E$ type the topological vertex cannot be used since the corresponding CalabiYau threefold is not toric. However, by using the fact that holomorphic curves in the geometry are in one to one correspondence with positive roots of the ADE groups we can generalize the partition function to all ADE cases to obtain

$$
\begin{aligned}
& Z_{G}=Z_{1}^{r+1}\left(\prod_{n=1}^{\infty}\left(1-Q_{\rho}^{n}\right)\right)^{r} \prod_{\alpha \in \Delta_{+}} \\
& \times \frac{G_{2}\left(Q_{\alpha} Q_{m} \sqrt{t q} ; \rho, \epsilon_{1},-\epsilon_{2}\right) G_{2}\left(Q_{\alpha} Q_{m}^{-1} \sqrt{t q} ; \rho, \epsilon_{1},-\epsilon_{2}\right)}{G_{2}\left(Q_{\alpha} t ; \rho, \epsilon_{1},-\epsilon_{2}\right) G_{2}\left(Q_{\alpha} q ; \rho, \epsilon_{1},-\epsilon_{2}\right)}
\end{aligned}
$$

where $r$ is the rank of the group $G$ and $\Delta_{+}$is the set of positive real roots corresponding affine ADE group. The parameters $Q_{\alpha}$ and $Q_{\rho}$ are related to the Kähler parameters of the resolved orbifold,

$Q_{\alpha}=e^{-\int_{C_{\alpha}} \omega}, \quad Q_{\rho}=e^{-\int_{C_{\delta}} \omega}$,

where $C_{\alpha}$ is the holomorphic curve corresponding to the positive real root $\alpha$ and $C_{\delta}$ is the holomorphic curve corresponding to the imaginary root $\delta$ of the affine ADE group. In the above equation $\omega$ is the complexified Kähler form of the resolved orbifold. Since the function $G_{2}$ itself is a product we see that $Z_{G}$ is an infinite product over positive roots of the affine $G$. Due to modular transformation properties of the function $G_{2}$ the partition function $Z_{G}$ satisfies a nonperturbative modular transformation $[5,14]$,

$Z_{G}\left(-\frac{1}{\rho}, \frac{\epsilon_{1}}{\rho}, \frac{\epsilon_{2}}{\rho}\right)=\frac{Z_{G}\left(\rho, \epsilon_{1}, \epsilon_{2}\right)}{Z_{G}\left(\frac{\rho}{\epsilon_{1}},-\frac{1}{\epsilon_{1}}, \frac{\epsilon_{2}}{\epsilon_{1}}\right) Z_{G}\left(\frac{\rho}{\epsilon_{2}}, \frac{\epsilon_{1}}{\epsilon_{2}},-\frac{1}{\epsilon_{2}}\right)}$.

From the partition function in Eq. (18) we can calculate the bound states by taking the plethystic logarithm,

$$
\begin{aligned}
F_{G}= & \mathrm{P} \log Z_{G} \\
= & (r+1) F_{1}+r \frac{Q_{\rho}\left(\sqrt{t q}+\frac{1}{\sqrt{t q}}-\sqrt{\frac{t}{q}}-\sqrt{\frac{q}{t}}\right)}{1-Q_{\rho}} \\
& +\sum_{\alpha \in \Delta_{+}} \frac{\left(Q_{\alpha}+Q_{\alpha}^{-1} Q_{\rho}\right)\left(Q_{m}+Q_{m}^{-1}-\sqrt{t q}-\frac{1}{\sqrt{q t}}\right)}{\left(1-Q_{\rho}\right)} .
\end{aligned}
$$


Thus we see that there is a single state corresponding to each positive root and there are no bound states corresponding to sum of positive roots. Actually bound states appear when we turn on the momentum along the compact direction. To see this recall that a dual description of this brane system is given by $N=1 * 5 \mathrm{D}$ affine ADE gauge theory. The partition function in Eq. (18) is the perturbative part of partition function of this gauge theory. Including the instanton part requires turning on momentum along the compact direction, i.e., going away from the $\tau \mapsto i \infty$ limit, since $\tau$ is the gauge coupling on the gauge theory side and equal to $1 / R$ where $R$ is the radius of the compact direction on which the M5-brane is wrapped. Thus if we consider instanton contribution the partition function of the gauge theory becomes

$\widehat{Z}_{G}=Z_{G}\left(1+Q_{\tau} I_{1}+\cdots\right)$,

where $I_{1}$ is the one instanton contribution. The one instanton moduli space can be factored,

One instanton moduli space $=\mathbb{C}^{2} \times \mathcal{M}_{1}$,

where the factor $\mathbb{C}^{2}$ gives the position of the instanton in $\mathbb{C}^{2}$ and $\mathcal{M}_{1}$ includes the orientation of the instanton within the gauge group. The Hilbert series of $\mathcal{M}_{1}$ is well known and given by the character of the adjoint representation of the ADE group $\chi_{G \text {,adj }}\left(Q_{i}\right)$ [15] and the contribution from the $\mathbb{C}^{2}$ factor is given by $\left(q^{1 / 2}-q^{-1 / 2}\right)^{-1}\left(t^{1 / 2}-t^{-1 / 2}\right)^{-1}$. Thus the partition function becomes

$\widehat{Z}_{G}=Z_{G}\left(1+Q_{\tau} \frac{\chi_{G, \text { adj }}\left(Q_{i}\right)}{\left(q^{\frac{1}{2}}-q^{-\frac{1}{2}}\right)\left(t^{\frac{1}{2}}-t^{-\frac{1}{2}}\right)}+\cdots\right)$.

Taking the plethystic logarithm to get the contribution of the bound states we get

$$
\begin{aligned}
\widehat{F}_{G} & =\operatorname{Plog} \widehat{Z}_{G} \\
& =F_{G}+Q_{\tau} \frac{\chi_{G, \text { adj }}\left(Q_{i}\right)}{\left(q^{\frac{1}{2}}-q^{-\frac{1}{2}}\right)\left(t^{\frac{1}{2}}-t^{-\frac{1}{2}}\right)}+\cdots .
\end{aligned}
$$

Since the factor $\chi_{G \text {,adj }}\left(Q_{i}\right)$ contains products of factors $Q_{i}$ corresponding to different simple roots therefore we see that we now have bound states corresponding to sum of positive roots. These bound states disappear in the limit $Q_{\tau} \mapsto 0$ and hence these states corresponding to simple roots form bound states only with the help of momentum on the compact circle.

\section{Conclusions}

In this short paper we discussed the partition function of an M5-brane with transverse orbifold. We saw that the partition function calculated using the topological vertex captures the modes of the tensor multiplet corresponding to the M5-brane as well as the modes of the 3-form field on the transverse orbifold. We generalized the partition function to the case of ADE orbifold and argued that bound states of M-strings corresponding to pair of simple roots appear when the M5 brane is wrapped on a circle and momentum is turned on the circle.

It will be useful to study the dual partition function coming from the worldvolume of the M2-branes for arbitrary ADE orbifold. In the case of A type orbifold the M2-brane theory in the infrared gives a $(0,2)$ sigma model whose target space is the product of Hilbert scheme of points on $\mathbb{C}^{2}$. For arbitrary ADE it is expected that the target space is a product of instanton moduli space with ranks equal to the Dynkin label of the corresponding ADE diagram [10].

Open Access This article is distributed under the terms of the Creative Commons Attribution 4.0 International License (http://creativecomm ons.org/licenses/by/4.0/), which permits unrestricted use, distribution, and reproduction in any medium, provided you give appropriate credit to the original author(s) and the source, provide a link to the Creative Commons license, and indicate if changes were made. Funded by $\mathrm{SCOAP}^{3}$.

\section{Appendix A}

We give some useful formulas in this appendix which have been used in paper. In the text Greek letters $\lambda, \mu, v$ have been used to denote partitions of natural numbers and the notation $(i, j) \in \lambda$ represents coordinates of a box in the Young diagram of the partition $\lambda$. The index $i$ labels the parts of the partition and goes from 1 to $\ell(\lambda)$, where $\ell(\lambda)$ is the number of parts of the partition. If we fix $i$ the index $j$ takes values in the set $\left\{1,2, \ldots, \lambda_{i}\right\}$ where $\lambda_{i}$ is the $i$ th part of the partition. $\lambda^{t}$ denotes the transpose of the partition which is obtained by reflection of the Young diagram of $\lambda$ in the diagonal.

The refined topological vertex is defined as $[7,16]$ :

$$
\begin{aligned}
& C_{\lambda \mu \nu}(t, q)=\left(\frac{q}{t}\right)^{\frac{\|\mu\|^{2}}{2}} t^{\frac{\kappa(\mu)}{2}} q^{\frac{\|v\|^{2}}{2}} \widetilde{Z}_{\nu}(t, q) \\
& \times \sum_{\eta}\left(\frac{q}{t}\right)^{\frac{|\eta|+|\lambda|-|\mu|}{2}} s_{\lambda^{t} / \eta}\left(t^{-\rho} q^{-\nu}\right) s_{\mu / \eta}\left(t^{-\nu^{t}} q^{-\rho}\right),
\end{aligned}
$$

where

$$
|\mu|=\sum_{i=1}^{\ell(\mu)} \mu_{i}, \quad\|\mu\|^{2}=\sum_{i=1}^{\ell(\mu)} \mu_{i}^{2},
$$

$\kappa(\mu)=\|\mu\|^{2}-\left\|\mu^{t}\right\|^{2}$,

and $\widetilde{Z}_{v}(t, q)$ is defined as

$$
\widetilde{Z}_{v}(t, q)=\prod_{(i, j) \in v}\left(1-q^{v_{i}-j} t^{\nu_{j}^{t}-i}\right)^{-1} .
$$


The functions $s_{\lambda / \eta}(\mathbf{x})$ are the skew-Schur functions defined as

$s_{\lambda / \eta}(\mathbf{x})=\sum_{\mu} N_{\eta \mu}^{\lambda} s_{\mu}(\mathbf{x})$,

where $s_{\lambda}(\mathbf{x})$ is the Schur function in the variables $\mathbf{x}=$ $\left\{x_{1}, x_{2}, \ldots\right\}$ and $N_{\eta \mu}^{\lambda}$ are the Littlewood-Richardson coefficients.

The refined vertex is a function of two parameters $q$ and $t$. For $q=t$ it reduces to the usual topological vertex [17] with $g_{s}=\ln q$ being the topological string coupling constant. The parameters $q$ and $t$ are related to the Omega background parameters $\left(\epsilon_{1}, \epsilon_{2}\right)$ [18] as follows:

$(q, t)=\left(e^{i \epsilon_{1}}, e^{-i \epsilon_{2}}\right)$,

so that the unrefined case corresponds to $\epsilon_{1}+\epsilon_{2}=0$.

\section{References}

1. B. Haghighat, C. Kozcaz, G. Lockhart, C. Vafa, Orbifolds of M-strings. Phys. Rev. D 89(4), 046003 (2014). doi:10.1103/ PhysRevD.89.046003. arXiv:1310.1185 [hep-th]

2. S. Hohenegger, A. Iqbal, M-strings, elliptic genera and $\mathcal{N}=4$ string amplitudes. Fortsch. Phys. 62, 155 (2014). doi:10.1002/prop. 201300035. arXiv:1310.1325 [hep-th]

3. S. Hohenegger, A. Iqbal, S.J. Rey, Instanton-monopole correspondence from M-branes on $\mathbb{S}^{1}$ and little string theory. Phys. Rev. D 93(6), 066016 (2016). doi:10.1103/PhysRevD.93.066016. arXiv:1511.02787 [hep-th]

4. L. Bhardwaj, M. Del Zotto, J.J. Heckman, D.R. Morrison, T. Rudelius, C. Vafa, F-theory and the classification of little strings. Phys. Rev. D 93(8), 086002 (2016). doi:10.1103/PhysRevD.93. 086002. arXiv:1511.05565 [hep-th]
5. G. Lockhart, C. Vafa, Superconformal partition functions and nonperturbative topological strings. arXiv:1210.5909 [hep-th]

6. N.C. Leung, C. Vafa, Branes and toric geometry. Adv. Theor. Math. Phys. 2, 91 (1998). arXiv:hep-th/9711013

7. A. Iqbal, C. Kozcaz, C. Vafa, The refined topological vertex. JHEP 0910, 069 (2009). arXiv:hep-th/0701156

8. A. Iqbal, B.A. Qureshi, K. Shabbir, M.A. Shehper, Brane webs and random processes. Int. J. Modern Phys. A 30(33), 1550202. arXiv:1509.06295 [hep-th]

9. H. Nakajima, Lectures on Hilbert schemes of points on surfaces, vol. 18. AMS University Lecture Series (1999)

10. K. Shabbir, Work in progress

11. P. Arvidsson, E. Flink, M. Henningson, The $(2,0)$ supersymmetric theory of tensor multiplets and self-dual strings in six dimensions. JHEP 05, 048 (2004)

12. B. Haghighat, A. Iqbal, C. Kozcaz, G. Lockhart, C. Vafa, M-strings. arXiv:1305.6322 [hep-th]

13. R. Gopakumar, C. Vafa, M theory and topological strings. 2. arXiv:hep-th/9812127

14. A. Iqbal, K. Shabbir, Elliptic CY3folds and non-perturbative modular transformation. Eur. Phys. J. C 76, 148 (2016). doi:10.1140/ epjc/s10052-016-4006-Z

15. S. Benvenuti, A. Hanany, N. Mekareeya, The Hilbert series of the one instanton moduli space. JHEP 1006, 100 (2010). doi:10.1007/ JHEP06(2010)100. arXiv:1005.3026 [hep-th]

16. T.J. Hollowood, A. Iqbal, C. Vafa, Matrix models, geometric engineering and elliptic genera. JHEP 03, 069 (2008). arXiv:hep-th/0310272

17. M. Aganagic, A. Klemm, M. Marino, C. Vafa, The topological vertex. Commun. Math. Phys. 254, 425-478 (2005). arXiv:hep-th/0305132

18. N.A. Nekrasov, Seiberg-Witten prepotential from instanton counting. Adv. Theor. Math. Phys. 7(5), 831 (2003). doi:10.4310/ATMP. 2003.v7.n5.a4. arXiv:hep-th/0206161 